Supporting Information

\title{
Effect of Nanoparticle Size on Plasmon-Driven Reaction Efficiency
}

\author{
Seokheon Kim, Sungwoon Lee, and Sangwoon Yoon* \\ Department of Chemistry, Chung-Ang University, 84 Heukseok-ro, Dongjak-gu, Seoul 06974, \\ Republic of Korea. \\ *E-mail: sangwoon@cau.ac.kr
}




\section{Comparison of Shape and Size Homogeneity for AuNPs and AuNSs}

(a) AuNPs

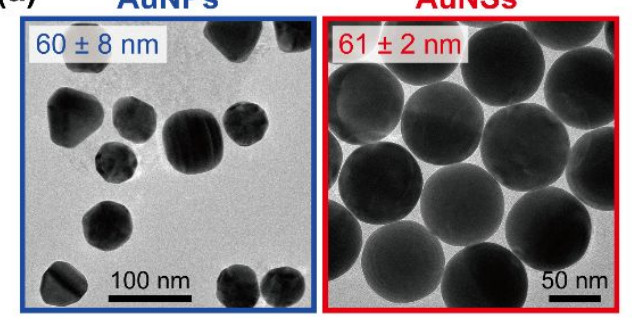

(b)

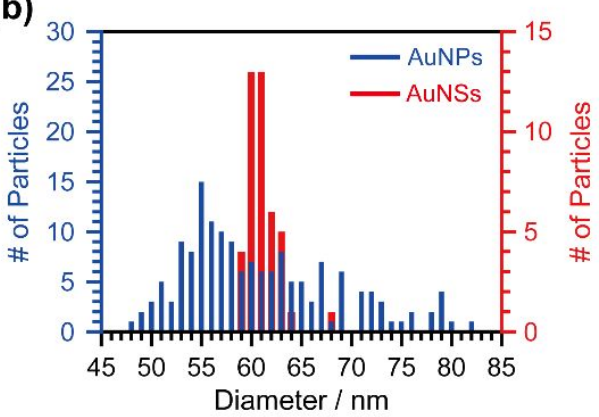

Figure S1. Comparison between AuNPs and AuNSs. (a) Representative TEM images of AuNPs and AuNSs with similar average diameters. (b) Size distributions of AuNPs and AuNSs. The AuNPs and AuNSs were prepared using the Puntes method and the Xia method, respectively. ${ }^{1,2}$

\section{Characteristics of Synthesized AuNSs}

Table S1. Structural and optical properties of synthesized AuNSs

\begin{tabular}{|c|c|c|c|c|c|c|c|c|}
\hline $\begin{array}{l}\text { Avg. Dia. } \\
\text { (nm) }\end{array}$ & $\begin{array}{c}\text { SD } \\
(\mathrm{nm})\end{array}$ & $\begin{array}{l}\text { RSD } \\
\text { (\%) }\end{array}$ & Sphericity ( $\pm S D$ ) & $\begin{array}{c}\lambda_{\text {LSPR }} \text { (expt) } \\
(\mathrm{nm})\end{array}$ & $\begin{array}{c}\lambda_{\text {LSPR }} \text { (calc) } \\
(\mathrm{nm})\end{array}$ & $\begin{array}{c}\Delta \lambda \\
(n m)\end{array}$ & $N$ & $\begin{array}{c}\zeta \text {-Potential } \\
(\mathrm{mV})\end{array}$ \\
\hline 26 & 1.5 & 5.8 & $0.90( \pm 0.05)$ & 523 & 526 & 3 & 308 & +61 \\
\hline 39 & 1.9 & 4.8 & $0.96( \pm 0.02)$ & 525 & 529 & 4 & 87 & +59 \\
\hline 61 & 1.6 & 2.6 & $0.96( \pm 0.01)$ & 530 & 539 & 9 & 42 & +40 \\
\hline 72 & 2.0 & 2.8 & $0.96( \pm 0.01)$ & 538 & 546 & 8 & 218 & +44 \\
\hline 84 & 2.8 & 3.4 & $0.95( \pm 0.03)$ & 543 & 555 & 12 & 127 & +30 \\
\hline 94 & 3.5 & 3.7 & $0.94( \pm 0.03)$ & 553 & 565 & 12 & 230 & N.A. \\
\hline 114 & 5.4 & 4.8 & $0.94( \pm 0.03)$ & 569 & 584 & 15 & 75 & +33 \\
\hline 133 & 9.4 & 7.1 & $0.93( \pm 0.04)$ & 586 & 609 & 23 & 200 & +32 \\
\hline
\end{tabular}




\section{Calculated Extinction, Absorption, and Scattering Spectra for AuNSs of Different Sizes}
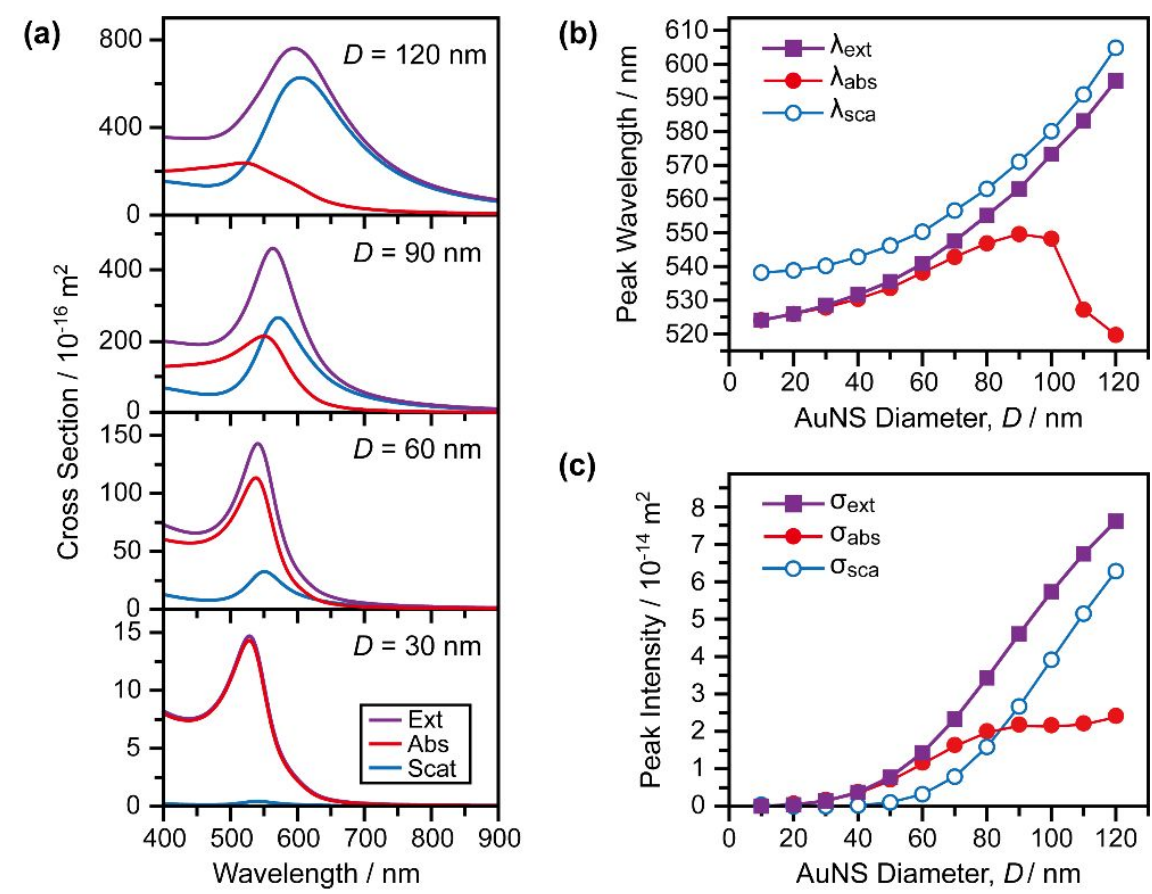

(c)

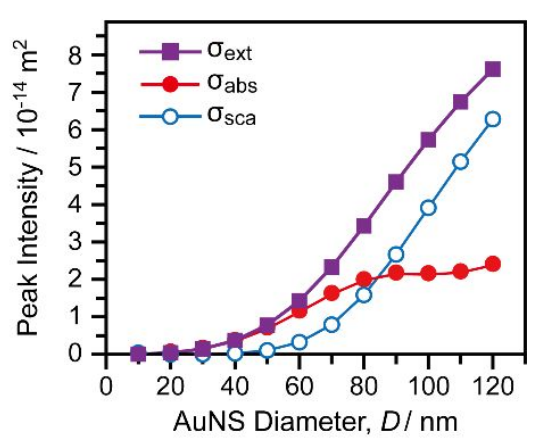

Figure S2. (a) Selected extinction (purple), absorption (red), and scattering (blue) spectra calculated for AuNSs with diameter D. (b) Peak wavelengths and (c) peak intensities in the spectra as a function of AuNS size.

\section{Hot Carrier Generation Efficiency per Photon as a Function of AuNS Size}

Since the plasmon-driven reaction yield is determined by the absorption of light and subsequent generation of hot carriers, dividing the experimental reaction yield by the absorption cross section leads to the hot carrier generation efficiency per absorbed photon. We plot the efficiency against AuNS size in Figure S3 below. The hot carrier generation efficiency decreases with the AuNS size, following the generally accepted electron-surface scattering rate $\left(\gamma_{e}\right.$-surface $)$. The decrease fits to the power function, $1 / D^{a}$ with $\mathrm{a}=1.77 \pm 0.28$ (blue line) the best. However, when we omit the point at $133 \mathrm{~nm}$ considering the large uncertainty in size as in Figure $2 b$, the quantum efficiency for hot carrier generation fits well to $1 / D$ (red line), in agreement with the Kreibig's equation. This result implies that synthesis of monodisperse perfect spherical AuNSs and accurate measurements of the reaction yield will reveal the precise relationship between the size of a AuNS and hot carrier generation efficiency upon plasmon excitation. 


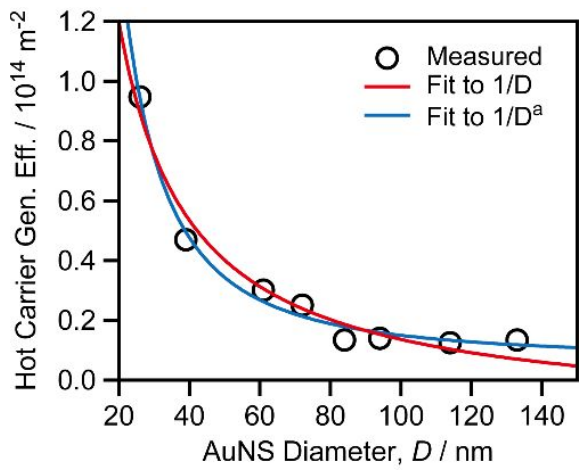

Figure S3. Hot carrier generation efficiency per absorbed photon as a function of AuNS size. The hot carrier generation efficiency per absorbed photon was obtained by dividing the experimental reaction yield by the calculated absorption cross section of the NPoM system at $785 \mathrm{~nm}$ for each size of AuNS (open circles). The efficiency was best fitted to $1 / \mathrm{D}^{\mathrm{a}}$ with $\mathrm{a}=1.77$ \pm 0.28 (blue line). When the point at $133 \mathrm{~nm}$ that has a large uncertainty in size is omitted, the fitting yields $a=1.14 \pm 0.26$, close to the Kreibig's term ( $a=1$, red line) for the electron-surface scattering.

\section{References}

1. Bastús, N. G.; Comenge, J.; Puntes, V. Kinetically Controlled Seeded Growth Synthesis of CitrateStabilized Gold Nanoparticles of up to 200 nm: Size Focusing Versus Ostwald Ripening. Langmuir 2011, 27, 11098-11105.

2. Zheng, Y.; Zhong, X.; Li, Z.; Xia, Y. Successive, Seed-Mediated Growth for the Synthesis of Single-Crystal Gold Nanospheres with Uniform Diameters Controlled in the Range of 5-150 nm. Part. Part. Syst. Charact. 2014, 31, 266-273. 